\title{
Modellierung der Reorientierung des Actin-Zytoskelettes in Endothel- zellen unter dem Einfluß von fluß-induzierten Scherspannungen
}

\author{
A. Suciu, G. Civelekoglu, Y. Tardy and J-J. Meister \\ Laboratoire de Génie Médical \\ Eidgenössisch Technische Hochschule Lausanne - Schweiz \\ LGM - PSE - Ecublens, EPFL, CH-1015 Lausanne
}

\section{EINLEITUNG :}

Die Übertragung von äußeren Kräften auf Endothelzellen führt zu signifikanten biochemischen und morphologischen Veränderungen [1]. Untersuchungen in-vivo und in-vitro demonstrieren im besonderen den Einfluß von fluß-induzierten Scherspannungen auf die Funktion und Erscheinungsform der Zellen [2]. Die Zellen verlängern und reorientieren sich in der Richtung der auf sie einwirkenden Blutströmung. Dieses Phänomen steht u.a. im Verdacht, in der Lokalisierung von arterioskeloritischen Plaques im Blutgefäßsystem involviert zu sein [3].

Die Änderung der äußeren morphologischen Form wird begleitet von einer Reorganisation des Zellskelettes im allgemeinen, und des F-Actin-Zytoskelettes im besonderen. Die einzelnen Actin-Filamente des grobmaschigen isotropen Netz-werkes, sowie vor allem die dominanten Actin-Stressfasern (stress fibers) reorientieren sich parallel zur langen Hauptachse der Zellen, und somit parallel zur Flußrichtung [4]. Die ursächlichen Mechanismen dieses Reorientierungsprozesses sind aber noch unklar und umstritten [5] und daher Gegenstand dieser Studie.

\section{MODELL:}

Angepasst an die Fragestellung berücksichtigt unser 2-dimensionales Modell des Actin-Zytoskelettes nur die winkelabhängige Verteilung der Actin-Filamente. Wir unterscheiden zwischen Filamenten, die mit TransmembranProteinen (Integrins) verbunden sind, und solchen, die es nicht sind. Unser Modell basiert weiterhin auf dem folgenden hypothetischen Mechanismus für die Übertragung der Scherspannungen auf die Actin-Filamente im Zellinneren. Die o.g. Transmembran-Proteine werden in der quasi-flüssigen Zellmembran unter dem Einfluß der flußinduzierten Scherspannungen lateral verschoben. Die Translation dieser Proteine führt zu einer Rotation der Actin-Filamente, die im Zellinneren über u.U. komplexe Proteinketten mit ihnen verbunden sind [6]. Wir kombinieren diesen Ansatz mit einer existierenden phänomenologischen Beschreibung der Dynamik von Actin-Filamenten und deren Bindungsproteinen [7]. Diese repräsentiert in Rahmen des Modells die permanente Neuorientierung von Actin-Filamenten im Zytoskelett aufgrund der dynamischen De- und Repolymerisation von Actin-Filamenten (Austauschvorgänge zwischen Actin-Monomeren und polymeren, auch turn-over genannt ), sowie die Formation der Actin-Stressfasern mit Hilfe der Bindungsproteine [8]. Auf diese Weise beschreiben wir die verschiedenen Kom- ponenten des Actin-Zytoskelettes und die berücksichtigten biophysikalischen Eigenschaften der ActinFilamente mit Hilfe eines Satzes von 4 gekoppelten Integro-Differential-Gleichungen.

\section{ERGEBNISSE:}

Die Resultate der numerischen Simulation der ModellGleichungen demonstrieren bei geeigneter Wahl der $\mathrm{Pa}$ rameter die Reorientierung des gesamten Actin-Zytoskelettes unter dem Einfluß der Scherspannungen. Dieses geschieht im Rahmen des Modells allein aufgrund der Dynamik der Actin-Filamente und ihrer molekularen Wechselwirkungen mit den Actin-Bindungsproteinen. Wir zeigen weiterhin die Abhängigkeit der Ausprägung des Phänomens von der Stärke der applizierten Scherspannung, in Übereinstimmung mit den experimentellen Beobachtungen.

\section{DISKUSSION:}

Wir stellen mit diesem Modell eine mögliche Erkiärung vor, für dieses seit langem bekannte, aber noch unverstandene komplexe biologische Phänomen. Das Modell basiert auf einigen wenigen biophysikalischen Elementen, und berücksichtigt nicht explizit zahlreiche bekannte biochemische Prozesse, die außerdem in diesem Zusammenhang eine wichtige Rolle spielen könnten. Deshalb schlagen wir weitere Experimente vor zur Bestätigung des Modells, bzw. zur Überprüfung der ihm zugrundeliegenden Hypothesen.

\section{LITERATUR:}

[1] Zhao, S., et al.; Arteriosclerosis, Thrombosis, and Vascular Biology.; 15(10) 1781-1786.(1995)

[2] Ando, J., et al.; Frontiers of Medical and Biological Engineering.; 5(4): 245 - 264.(1993)

[3] Ku, D. N., et al.; Arteriosclerosis.; 5(3): 293 - 302 (1985)

[4] Wechezak, A. R., et al.; Laboratory Investigation.; 53: 639 - 647.(1985)

[5] Davies, P. F., et al.; Circulation Research.; 72(2): 239 - 245.(1993)

[6] Kusumi, A., et al.; Biophysical Joumal.; 65: 2021 2040.(1993)

[7] Civelekoglu, G., et al.; Bull. Math. Biol.; 56(4): 587616.(1994)

[8] Pollard, T. D., et al.; Annual Review of Biochemistry.; 55: 987 - 1035.(1986) 